\title{
Vacunación programática 2020 en Chile en tiempos de pandemia por SARS-CoV-2
}

\section{Routine immunization 2020 in Chile during the SARS-CoV-2 pandemic}

\author{
Magdalena Bastías ${ }^{1}$, Iván Brstilo y Cecilia González
}

'Departamento de Inmunizaciones, Ministerio de Salud de Chile.

Los autores declaran no haber recibido financiamiento para la realización de este trabajo.

Recibido: 5 de abril de 2021 / Aceptado: 8 de abril de 2021

\section{Resumen}

En marzo de 2020, la Organización Mundial de la Salud (OMS) hizo un llamado a mantener la vacunación programática siempre que se pudieran garantizar condiciones seguras frente a la circulación de SARS-CoV-2. Asimismo, advirtió sobre el riesgo de interrupción de la vacunación programática secundaria a la pandemia por SARS-CoV-2. La interrupción de la vacunación genera un aumento en el número de personas susceptibles a las enfermedades que se busca prevenir y, así, de la probabilidad de uno o más brotes epidémicos. Estos, junto con incrementar la morbilidad y mortalidad en los grupos de riesgo, significarían una carga adicional al ya demandado sistema de salud por COVID-19. En agosto 2020, la OMS reportó interrupción de los servicios de salud esenciales en distintos países a raíz de la pandemia de SARS-CoV-2, afectando con mayor frecuencia a la vacunación de rutina y extramural. En Chile, la vacunación programática durante el 2020 disminuyó respecto del promedio del período 2015-2019, con la menor caída en la vacunación de los 2 meses de edad con hexavalente $(0,39 \%)$ y la mayor a los 18 meses también con hexavalente $(12,02 \%)$. La excepción fue la vacunación antineumocócica del adulto, con un incremento de $0,8 \%$ respecto del período 2015-2019.

Palabras clave: calendario nacional de inmunización; vacunación de rutina; cobertura de vacunación; pandemia por el nuevo coronavirus 2019-nCov; SARS-CoV-2; Chile.

\section{Introducción}

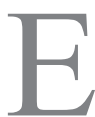
$\mathrm{n}$ los "Principios rectores para las actividades de inmunización durante la pandemia por COVID-19", la Organización Mundial de la Salud (OMS) hizo un llamado a los países a mantener la vacunación programática siempre que pudieran garantizar condiciones

\section{Abstract}

In March 2020, the World Health Organization (WHO) recommended that vaccination not be interrupted as long as countries could safely maintain this service in the context of the SARS-CoV-2 pandemic. WHO also warned about the risk of interruption of programmatic vaccination that generates an increase in the number of people susceptible to infections that are sought to be prevented with the use of vaccines and, therefore, an increase in the probability of disease outbreak. Along with increased morbidity and mortality in risk groups, vaccine-preventable disease outbreaks would put additional demand on the health system already burdened by COVID-19. In August 2020, WHO reported the interruption of essential health services in different countries as a result of the SARS-CoV-2 pandemic, which mainly affected routine and extramural vaccination. In Chile, routine vaccination coverage decreased during 2020 compared to the average coverage for the 2015-2019 period. The smallest decline was $0.39 \%$ for the hexavalent vaccine at 2 months of age and the largest decrease was $12.02 \%$ at 18 months for the same vaccine. The exception to the fall in coverage in 2020 was adult pneumococcal vaccination, which showed an increase of $0.8 \%$ compared to 2015-2019.

Keywords: immunization programs; vaccination coverage; 2019 novel coronavirus pandemic; SARS-CoV-2; pandemic. 
a las enfermedades que se busca prevenir y, por lo tanto, de la probabilidad de uno o más brotes epidémicos ${ }^{1}$. Estos, además de incrementar la morbilidad y mortalidad en los grupos de riesgo, significarían una carga adicional al ya exigido sistema de salud por COVID-19.

Si bien se entiende que los recursos humanos y materiales deben ser redistribuidos para dar respuesta a las necesidades de salud derivadas de la pandemia, es necesario asegurar que la respuesta a COVID-19 se dé en equilibrio con otras necesidades de salud que también significan alta morbilidad y mortalidad para grupos de riesgo $^{2}$. Sin embargo, este no ha sido el caso en todos los países. De acuerdo a la encuesta sobre servicios de salud esenciales durante la pandemia de SARS-CoV-2 realizada por la OMS, de 105 países participantes, 90\% reportó sufrir interrupción de servicios a raíz de la pandemia. Entre ellos, la mayor frecuencia de interrupción la sufría la vacunación programática y extramural (70\%), seguida del diagnóstico y tratamiento de enfermedades no transmisibles (69\%) y de la planificación familiar $(68 \%)^{3}$.

Durante el 2020, los servicios de vacunación infantil programática fueron interrumpidos a nivel mundial de una manera nunca antes vista desde la implementación de los programas ampliados de inmunización en la década de $1970^{4}$. Entre las razones para la interrupción de la vacunación, estuvieron: i) la resistencia por parte de los padres a salir de casa debido a las restricciones de movilidad para la prevención y control de SARS-CoV-2 o al temor a infectarse; ii) la disminución de la disponibilidad de trabajadores de la salud por restricciones de traslado, reasignación de sus jornadas a tareas de respuesta a COVID-19 o por falta de elementos de protección personal; iii) la migración interna para pasar la cuarentena en el pueblo de origen o zonas rurales, incrementando la inasistencia a las citas de vacunación ${ }^{2,5}$.

De acuerdo a información recolectada durante marzo y abril de 2020 por la OMS, el Fondo de las Naciones Unidas para la Infancia (UNICEF), Global Alliance for Vaccines and Immunisation (GAVI) y Sabin Institute, más de 60 países reportaron interrupciones moderadas o graves de la vacunación o una suspensión total de los servicios de vacunación, y se estimó que al menos 24 millones de personas en 21 países de bajos ingresos podrían haber perdido la oportunidad de vacunarse contra poliomielitis, sarampión, fiebre tifoidea, fiebre amarilla, cólera, rotavirus, virus papiloma humano (VPH), enfermedad meningocócica serogrupo A y rubéola debido a las interrupciones. Específicamente en sarampión y poliomielitis, las campañas de vacunación se suspendieron en 27 y 38 países, respectivamente ${ }^{5}$.

En Chile, el Decreto Exento $N^{\circ} 6$ del 29 de enero de 2010 y sus modificaciones posteriores disponen la vacunación obligatoria de la población contra hepatitis $\mathrm{B}$, hepatitis A, difteria, tétanos, tos convulsiva, poliomielitis, sarampión, rubéola, parotiditis, infecciones por virus del papiloma humano, y enfermedades invasoras por $M$. tuberculosis, $N$. meningitidis, $S$. pneumoniae y $H$. influenzae tipo b $(\mathrm{Hib})^{6,7}$. En contexto de pandemia de COVID-19 y manteniendo la seguridad del personal de salud y usuarios de los servicios de inmunización frente a la circulación de SARS-CoV-2, el Departamento de Inmunizaciones del Ministerio de Salud de Chile continuó ofreciendo la vacunación programática -vacunación del lactante, escolar y del adulto- según lo planificado inicialmente para el 2020.

\section{Objetivo}

Describir el desempeño de la vacunación programática de Chile 2020 en contexto de pandemia por SARS-CoV-2 respecto del período 2015-2019.

\section{Material y Métodos}

Descripción del avance, cobertura y refuerzo de la vacunación programática 2020 del niño y del adulto para la prevención de hepatitis $\mathrm{A}$, hepatitis $\mathrm{B}$, difteria, tétanos, tos convulsiva (pertussis), poliomielitis, sarampión, rubéola, parotiditis, infecciones por virus papiloma humano (VPH), enfermedades invasoras por $N$. meningitidis, $S$. pneumoniae y $H$. influenzae tipo b (Hib) y su comparación con el promedio de avance, cobertura y refuerzo del período 2015-2019. Las comparaciones de avance, cobertura y refuerzo se evaluaron con la prueba z. Se excluyeron las vacunas del recién nacido por ser administradas en forma intrahospitalaria, vacuna VPH en varones por haber sido introducida el 2019, vacuna contra varicela por haber sido introducida el 2020 y vacuna contra fiebre amarilla por ser programática exclusivamente en Rapa Nui.

Se definió avance de vacunación como la proporción de población objetivo que recibió la primera o segunda dosis de la vacuna recomendada a una edad específica al cierre del año calendario. En este estudio, la definición aplica a la vacunación con vacuna pentavalente (hepatitis $\mathrm{B}$, difteria, tétanos, pertussis, Hib) o hexavalente (hepatitis $\mathrm{B}$, difteria, tétanos, pertussis, Hib, poliomielitis) y con neumocócica conjugada a los 2 y 4 meses y con vacuna VPH en $4^{\circ}$ básico.

Cobertura de vacunación se definió como la proporción de población objetivo que, al cierre del año calendario, completó el esquema de vacunación recomendado para una edad específica. En este estudio, la definición aplica a la vacuna pentavalente o hexavalente a los 6 meses, vacuna neumocócica conjugada, sarampiónrubéola-parotiditis (SRP) y meningocócica a los 12 meses, vacuna hepatitis A de los 18 meses, vacuna VPH en niñas de $5^{\circ}$ básico, vacuna difteria, tétanos y pertussis acelular (dTpa) de la mujer embarazada a partir de la semana $28 \mathrm{de}$ 
gestación y vacuna neumocócica polisacárida a personas de 65 años y más.

Refuerzo se definió como la proporción de población objetivo que recibió una primera o segunda dosis de refuerzo de la vacuna recomendada a una edad específica al cierre del año calendario. En este estudio, la definición aplica a la vacuna hexavalente de los 18 meses (primer refuerzo), a las vacunas SRP y dTpa en $1^{\circ}$ básico (primer refuerzo) y a la vacuna dTpa en $8^{\circ}$ básico (segundo refuerzo).

\section{Resultados}

Con la excepción de la vacunación neumocócica del adulto, comparado con el promedio de avance o de cobertura de los años 2015 a 2019 según corresponda, la demanda de la vacunación programática del año 2020 disminuyó para todas las vacunas y en todas las citas de vacunación de manera heterogénea (Figura 1). En el lactante, la menor caída fue $0,39 \%$ en el avance de vacunación con hexavalente a los 2 meses de edad, en tanto las mayores caídas se presentaron en la edad de 18 meses, con un valor máximo de $12,02 \%$ de disminución en el refuerzo con la vacuna hexavalente y de $10,7 \%$ en la cobertura de la vacuna hepatitis A (Tabla 1). En el lactante, la demanda por las distintas vacunas recomendadas para una misma edad fue diferenciada, particularmente a los 12 meses, edad en que la demanda fue mayor para la vacunación SRP y menor para la antineumocócica, con una diferencia entre ellas de alrededor de 5 puntos porcentuales.

La vacunación escolar, que se realiza durante el segundo semestre de cada año y que para el 2020 consistió de la vacunación contra difteria, tétanos y pertussis acelular (dTpa) en $1^{\circ}$ y $8^{\circ}$ básico y contra VPH en $4^{\circ}$ y $5^{\circ}$ básico, la mayor caída durante el 2020 se registró en la dTpa de $8^{\circ}$ básico, con un $8,8 \%$ menos que el promedio del período 2015-2019. La segunda mayor caída en la vacunación escolar fue para la primera dosis de VPH ( $4^{\circ}$ básico), con $7,6 \%$ menos que años anteriores (Tabla 1 ).

El año 2020, la cobertura de vacunación neumocócica del adulto presentó $0,8 \%$ más que el promedio de cobertura de vacunación del período 2015-2019. Si bien la Tabla 1 muestra la cobertura de vacunación neumocócica del adulto al año de vacunación, la vacunación programática del adulto ofrece la oportunidad de estimar una cobertura dinámica, es decir, una en base al comportamiento de cada cohorte de nacimiento que va cumpliendo 65 años, más todos aquellos que no se vacunaron a esa edad y que adhieren después, por lo que su proyección es al aumento.

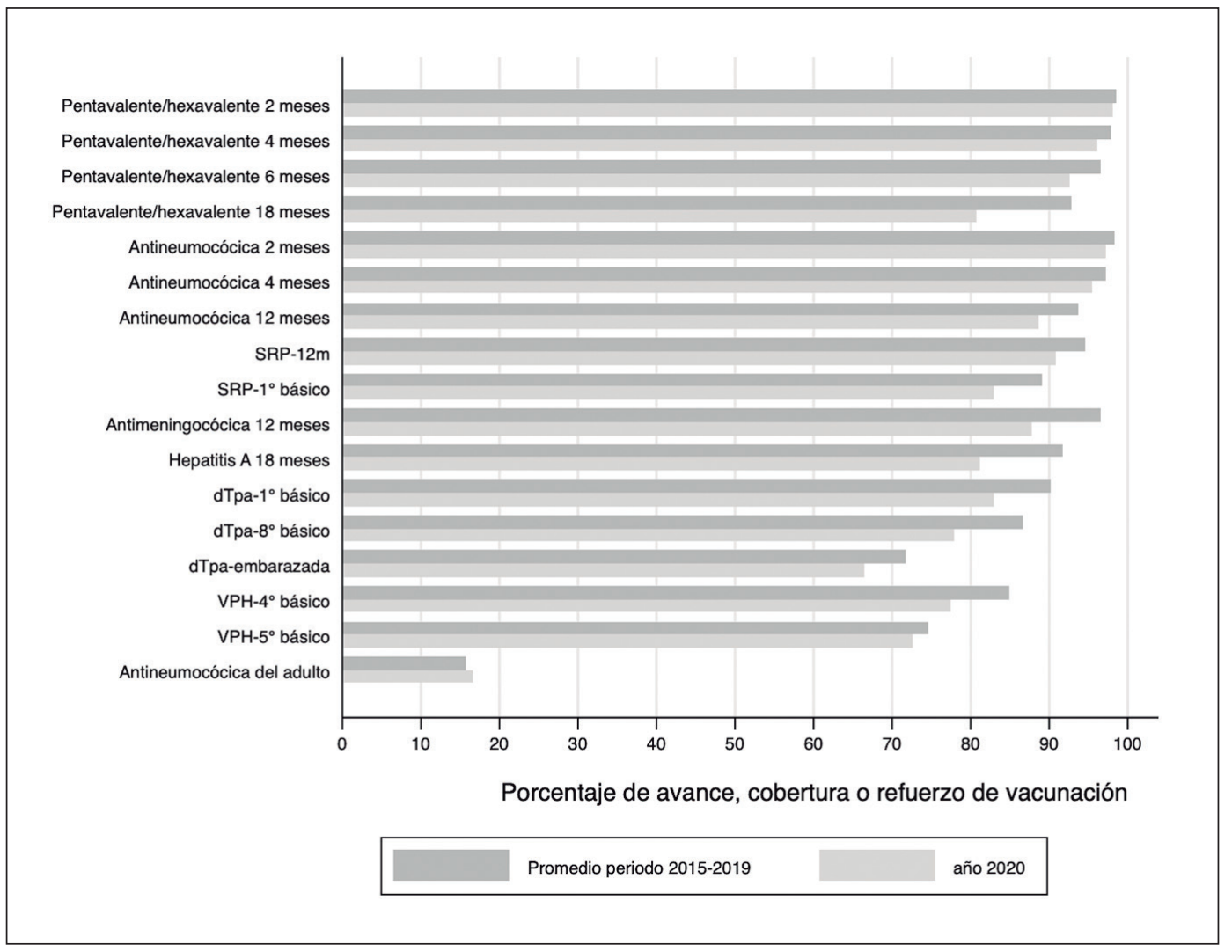

Figura 1. Avance, cobertura y refuerzo de vacunación programática 2015-2020 en Chile según vacuna y cita de vacunación. 


\begin{tabular}{|c|c|c|c|c|c|c|c|c|c|c|}
\hline \multirow[t]{2}{*}{ Vacuna } & \multirow[t]{2}{*}{ Edad o grupo de riesgo } & \multicolumn{6}{|c|}{ Cobertura anual de vacunación (\%) } & \multirow{2}{*}{$\begin{array}{c}\bar{x}(2015- \\
2019)-2020^{*}\end{array}$} & \multirow[t]{2}{*}{$\mathrm{IC}_{95 \%}$} & \multirow[t]{2}{*}{ p } \\
\hline & & 2015 & 2016 & 2017 & 2018 & 2019 & 2020 & & & \\
\hline \multirow{4}{*}{$\begin{array}{l}\text { Pentavalente o } \\
\text { hexavalente }\end{array}$} & 2 meses & 97,9 & 98,2 & 98,6 & 99,0 & 99,0 & 98,1 & 0,39 & $0,34-0,46$ & $<0,000$ \\
\hline & 4 meses & 97,1 & 98,1 & 97,8 & 98,3 & 98,4 & 96,2 & 1,80 & $1,72-1,89$ & $<0,000$ \\
\hline & 6 meses & 95,8 & 96,1 & 96,9 & 97,4 & 97,2 & 92,7 & 3,96 & $3,85-4,09$ & $<0,000$ \\
\hline & 18 meses & 91,2 & 95,8 & 92,7 & 93,0 & 91,7 & 80,9 & 12,02 & $11,88-12,22$ & $<0,000$ \\
\hline \multirow[t]{3}{*}{ Neumocócica conjugada } & 2 meses & 98,0 & 98,1 & 98,8 & 98,8 & 98,5 & 97,4 & 0,98 & $0,91-1,06$ & $<0,000$ \\
\hline & 4 meses & 96,7 & 96,7 & 97,7 & 98,0 & 97,9 & 95,6 & 1,73 & $1,64-1,82$ & $<0,000$ \\
\hline & 12 meses & 90,5 & 92,9 & 93,8 & 95,7 & 95,8 & 88,8 & 4,88 & $4,74-5,02$ & $<0,000$ \\
\hline \multirow{2}{*}{$\begin{array}{l}\text { Sarampión, rubéola y } \\
\text { parotiditis }\end{array}$} & 12 meses & 91,5 & 94,7 & 96,1 & 96,0 & 95,4 & 90,9 & 3,78 & $3,65-3,91$ & $<0,000$ \\
\hline & $1^{\circ}$ básico & 88,8 & 87,1 & 86,2 & 93,1 & 90,7 & 83,1 & 6,03 & $5,87-6,19$ & $<0,000$ \\
\hline Meningocócica conjugada & 12 meses & 95,4 & 95,9 & 97,5 & 97,1 & 97 & 87,8 & 8,74 & $8,61-8,89$ & $<0,000$ \\
\hline Hepatitis A & 18 meses & * & * & * & 91,2 & 92,5 & 81,2 & 10,71 & $10,52-10,89$ & $<0,000$ \\
\hline \multirow{3}{*}{$\begin{array}{l}\text { Difteria, tétanos y } \\
\text { pertussis acelular }\end{array}$} & $1^{\circ}$ básico & 88,1 & 87,6 & 90,8 & 92,6 & 92,7 & 83,0 & 7,34 & $7,18-7,50$ & $<0,000$ \\
\hline & $8^{\circ}$ básico & 83,9 & 81,9 & 87,9 & 90,0 & 90,0 & 77,9 & 8,75 & $8,57-8,91$ & $<0,000$ \\
\hline & Embarazadas $\geq 28$ semanas gestación & * & * & 75,5 & 70,6 & 69,4 & 66,5 & 4,34 & $4,11-4,57$ & $<0,000$ \\
\hline \multirow[t]{2}{*}{ Virus papiloma humano $^{+}$} & $4^{\circ}$ básico & 85,0 & 77,0 & 83,0 & 87,6 & 92,2 & 77,6 & 7,61 & $7,36-7,85$ & $<0,000$ \\
\hline & $5^{\circ}$ básico & 76,0 & 73,0 & 70,0 & 75,9 & 79,1 & 72,7 & 2,35 & $2,08-2,62$ & $<0,000$ \\
\hline Neumocócica polisacárida & Personas de 65 y más años & 15,2 & 12,9 & 13,8 & 17,3 & 19,5 & 16,7 & $-0,80$ & $-0,61--0,99$ & $<0,000$ \\
\hline
\end{tabular}

Así, las coberturas dinámicas para la vacunación neumocócica del adulto son de 68,4\% para el año 2015 (cohorte de nacidos el año 1950), 60\% para el 2016 (cohorte 1951), $56,7 \%$ para el 2017 (cohorte 1952), 53,9\% para el 2018 (cohorte 1953), 40,6\% para el 2019 (cohorte 1954) y $18,1 \%$ en lo que va de la cohorte del 1955 que el año 2020 cumplió 65 años (más todos aquellos mayores 65 años que se vacunaron el 2020). En el período 2015-2020, a la fecha de elaboración de este trabajo, el $72 \%$ de las personas de 65 y más años en Chile se ha vacunado contra neumococo (estimación bruta).

\section{Discusión y Conclusiones}

El año 2020, los avances, coberturas y refuerzos de vacunación programática en Chile disminuyeron respecto de años anteriores; esto en un contexto de alerta sanitaria a raíz de la pandemia causada por el virus SARS-CoV-2 para la que se han descrito razones para la interrupción de la vacunación, que son la resistencia a salir de casa debido al temor de infectarse y a las restricciones de movilidad para la prevención y control de SARS-CoV-2, la migración interna para pasar la cuarentena con familiares o en zonas rurales, y la disminución en la disponibilidad de trabajadores de la salud por restricciones de movilidad, por reasignación de sus jornadas a tareas de respuesta a COVID-19 o por falta de elementos de protección personal. En el caso de Chile, la vacunación programática no sufrió de reducciones de personal de salud.

Las bajas en la vacunación programática en Chile fluctuaron entre $0,39 \%$ y $1,80 \%$ en los avances, entre $3,96 \%$ y $10,71 \%$ en coberturas y entre $3,94 \%$ y $12,02 \%$ en los refuerzos. El 2020 hubo diferencias en la demanda de las distintas vacunas recomendadas para la misma edad, 
que, en base al actual registro y plan de uso de la vacuna neumocócica polisacárida en Chile (una dosis a los 65 años y más), la pregunta de interés es ¿qué proporción de la población mayor de 65 años está actualmente protegida con la vacuna antineumocócica? En términos brutos, esta proporción es de $72 \%$ en el país.

En Francia, las caídas en la vacunación infantil durante el confinamiento del 17 de marzo al 11 de mayo de 2020 fueron de $35 \%$ en la entrega de las vacunas pentavalente y hexavalente, de $43 \%$ en la vacunación SRP y $67 \%$ en la vacuna contra virus del papiloma humano (VPH), junto con una disminución de $71 \%$ en la venta de vacuna antitetánica9 . En Asia, Vietnam suspendió la vacunación programática entre el 1 y 15 de abril de 2020, India también la suspendió por reasignación de los recursos humanos a la respuesta sanitaria a COVID-19, y Pakistán, siguiendo la recomendación de la Iniciativa Global de Erradicación de Polio y donde la poliomielitis es endémica, pospuso la campaña de vacunación de puesta al día contra la polio ${ }^{10}$, además de experimentar una disminución de 52,5\% en el promedio diario de vacunaciones, registrando la mayor caída en la vacunación contra enfermedad invasora por $M$. tuberculosis (BCG), un 40,6\% ${ }^{11}$. Días después de la declaración de alerta sanitaria en Estados Unidos de América (E.U.A.) y comparado con semanas del mismo período del año anterior (enero-abril), los registros del programa federal de vacunas (VFC) muestran la caída en la solicitud y administración de vacunas programáticas del niño, excluyendo influenza. En vacunas contra sarampión, por ejemplo, las dosis administradas disminuyeron alrededor de $50 \%$ en niños de 24 meses o menos y en aproximadamente $75 \%$ en niños mayores de 24 meses hasta 18 años ${ }^{12}$. En otro período de estudio, enero-julio de 2019 versus 2020, y en base a información proveniente del sistema de seguro Medicare de E.U.A., la vacunación diaria de la vacuna neumocócica conjugada 13-valente, neumocócica polisacárida 23-valente, difteria-tétanos o difteria-tétanospertussis acelular y vacuna recombinante contra el herpes zoster disminuyó hasta $89 \%$ a mediados de marzo de 2020 , mostrando recuperación gradual a partir de junio de 2020, aunque siempre bajo lo observado en el mismo período de $2019^{13}$. En Michigan, E.U.A., al mes de mayo de 2020 respecto del mismo mes en año anteriores, y a excepción de la vacunación del recién nacido que se lleva a cabo en el hospital, la cobertura de las vacunas recomendadas para los 5 meses de edad disminuyó 18 puntos porcentuales y a los 16 meses, la vacunación contra sarampión cayó 5 puntos porcentuales. En el mismo Estado, entre enero y abril de 2020, la vacunación general de lactantes disminuyó 15,5\% y la vacunación de menores de 19 años, 21,5\%, ambas excluyendo la vacunación contra influenza ${ }^{14}$. En Inglaterra, tres semanas después de la introducción de la medida de distanciamiento físico, la administración de vacuna hexavalente disminuyó $6,7 \%\left(\mathrm{IC}_{95 \%}-7,1 ;-6,2\right)$ y 
la vacunación contra sarampión, 19,8\% (IC95\% -20,7 ; $-18,9)$ respecto del mismo momento del año anterior ${ }^{15}$. En África, el promedio de niños vacunados con esquema completo contra difteria, tétanos y parotiditis (DPT3) el segundo trimestre mostró la mayor caída respecto del primer trimestre en Guinea (52\%) y la menor, en Kenia y Ruanda ( $2 \%)$, en tanto la vacunación contra sarampión tuvo la mayor caída en Guinea (53\%) y la menor en la República Centroafricana $(3 \%)^{16}$.

Si bien la vacunación programática en Chile experimentó una caída durante el 2020 , año en alerta sanitaria por pandemia de SARS-CoV-2 y bajo medidas de control y prevención del nuevo coronavirus conocidas por afectar la demanda de la vacunación, tales como cuarentenas y distanciamiento físico, las bajas no superaron el $12 \%$ en el peor escenario, cifra que resulta inferior a lo reportado por otros países, aunque los períodos de análisis de coberturas durante la pandemia no resultan comparables entre ellos. Para el Departamento de Inmunizaciones de Chile, las caídas en las coberturas y refuerzos de vacunación influyeron tempranamente en el diseño y planificación de estrategias para la puesta al día de la vacunación en cada edad, entre ellas, el refuerzo de la comunicación con la red de salud a nivel medio y ejecutor, proporcionándoles guías y orientaciones y la oportuna difusión de instrucciones y normas ${ }^{17-19}$, junto con la capacitación permanente (online) y resolución de consultas y solicitudes. El buen desempeño de la vacunación programática en Chile 2020 en contexto de pandemia por SARS-CoV-2 obedece a la capacidad del Departamento de Inmunizaciones de responder oportunamente a crisis y demandas extraordinarias, como han sido los manejos de brotes epidémicos, de riesgos de exposición a infecciones secundarios a desastres naturales y, la más reciente, la replanificación de la Campaña Influenza 2020 debido a nuevas necesidades derivadas de la pandemia por SARS-CoV- ${ }^{20}$. La experiencia de vacunación poblacional acumulada por el Departamento de Inmunizaciones en conjunto con las SEREMI y los centros de atención primaria de salud le ha permitido a este sistema de inmunizaciones de Chile continuar procurando el acceso garantizado de la población a la vacunación estatal gratuita incluso en contexto de pandemia.

Agradecimientos: Manuscrito revisado por Sylvia Santander Rigollet, Jefa de la División de Prevención y Control de Enfermedades, Subsecretaría de Salud Pública, Ministerio de Salud de Chile.

\section{Referencias bibliográficas}

1.- Organización Mundial de la Salud. Principios rectores para las actividades de inmunización durante la pandemia de COVID-19 [Internet]. 2020. Available from: https://apps.who.int/iris/ handle/10665/331670.

2.- Nelson R. COVID-19 disrupts vaccine delivery. Lancet Infect Dis. 2020; 20 (5): 546. doi: 10.1016/S1473-3099(20)30304-2.

3.- World Health Organization. In WHO global pulse survey, $90 \%$ of countries report disruptions to essential health services since COVID-19 pandemic [Internet]. 2020. Available from: https://www.who.int/news/ item/31-08-2020-in-who-global-pulse-survey90-of-countries-report-disruptions-to-essentialhealth-services-since-covid-19-pandemic

4.- $\quad$ Torner N. Collateral effects of COVID-19 pandemic emergency response on worldwide immunizations. Vacunas. 21 (2): 73-5. doi: 10.1016/j.vacun.2020.07.002.

5.- World Health Organization. At least 80 million children under one at risk of diseases such as diphtheria, measles and polio as COVID-19 disrupts routine vaccination efforts, warn Gavi, WHO and UNICEF [Internet]. Available from: https://www.who. int/news/item/22-05-2020-at-least-80-millionchildren-under-one-at-risk-of-diseases-suchas-diphtheria-measles-and-polio-as-covid-19- disrupts-routine-vaccination-efforts-warn-gaviwho-and-unicef

6.- Subsecretaría de Salud Pública-Ministerio de Salud de Chile. Decreto Exento No 6 del 29 de enero 2010- Dispone la vacunación obligatoria contra enfermedades inmuno prevenibles de la población del país [Internet]. 2010. Available from: https://www.ben.cl/leychile/ navegar?idNorma $=1012507$

7.- Biblioteca del Congreso Nacional de Chile/ $\mathrm{BCN}$. Ley Chile [Internet]. Concordancias Decreto 6 Extento. 2020. Available from: https://www.bcn.cl/leychile/consulta/ vinculaciones/concordancia?idNorma $=101$ $2507 \&$ fechaVigencia $=2020-08-03 \&$ clase vinculacion $=$ CONCORDANCIA.

8.- Burgos P, Castro I, González C. Cobertura y oportunidad de vacunación contra difteriatétanos-pertussis en Chile, 2013-2017; un análisis de Kaplan-Meier utilizando el Registro Nacional de Inmunizaciones (póster congreso SOCHINF 2020). Rev Chilena Infectol 2020; 37 (s1): 33 .

9.- Billon-Denis E, Tournier J. COVID-19 et vaccination: une dérégulation globale. Med Sci (Paris) 2020; 36 (11): 1034-7. doi: 10.1051/ medsci/2020203.

10.- Dinleyici E, Borrow R, Palazzi M, van Damme $\mathrm{P}$, Munoz F. Vaccines and routine immunization strategies during the COVID-19 pandemic. Hum Vaccin Immunother. 2020; 17 (2): 400-7. doi: 10.1080/21645515.2020.1804776.

11.- Chandir S, Siddiqi D, Mehmood M, Setayesh $\mathrm{H}$, Siddique M, Mirza A. Impact of COVID-19 pandemic response on uptake of routine immunizations in Sindh, Pakistan: An analysis of provincial electronic immunization registry data. Vaccine. 2020; 38 (45): 7146-55. doi: 10.1016/j.vaccine.2020.08.019.

12.- Santoli J, Lindley M, DeSilva M, Kharbanda E, Daley M, Galloway L, et al. Effects of the COVID-19 pandemic on routine pediatric vaccine ordering and administration - United States, 2020. MMWR Morb Mortal Wkly Rep. 2020; 69 (19): 591-3. doi: 10.15585/mmwr. $\mathrm{mm} 6919 \mathrm{e} 2$.

13.- Hong K, Zhou F, Tsai Y, Jatlaoui T, Acosta A, Dooling K. Decline in receipt of vaccines by medicare beneficiaries during the COVID-19 Pandemic - United States, 2020. MMWR Morb Mortal Wkly Rep. 2021; 70 (7): 245-9. http:// dx.doi.org/10.15585/mmwr.mm7007a4 .

14.- Bramer C, Kimmins L, Swanson R, Kuo J, Vranesich P, Jacques-Carroll L, et al. Decline in child vaccination coverage during the COVID-19 pandemic - Michigan care improvement registry, May 2016-May 2020. MMWR Morb Mortal Wky Rep. 2020; 69 (20): 630-1. doi: 10.15585/mmwr.mm6920e1.

15.- McDonald H, Tessier E, White J, Woodruff M, Knowles C, Bates C, et al. Early impact of the coronavirus disease (COVID-19) 
la pandemia COVID-19 [Internet]. 2020.

pandemic and physical distancing measures on routine childhood vaccinations in England, January to April 2020. Euro Surveill. 2020; 25. doi: 10.2807/1560-7917. ES.2020.25.19.2000848.

16.- Masresha B, Luce R, Shibeshi M, Ntsama B, N'Diaye A, Chakauya J, et al. The performance of routine immunization in selected African countries during the first six months of the COVID-19 pandemic. Pan Afr Med J. 2020; 37 (Suppl 1): 12. doi: 10.11604/pamj. supp.2020.37.12.26107.

17.- Subsecretaría de Salud Pública-Ministerio de Salud de Chile. Ordinario B27 No 2148 12 de junio de 2020 Recomendaciones para la vacunación de recién nacidos durante
Available from: https://vacunas.minsal.cl/ wp-content/uploads/2020/07/Ord-B27-N ${ }^{\circ}$ 2148-Recomendaciones-para-la-vacunacion-derecien-nacidos-durante-la-pandemia-covid1912-junio-2020.pdf.

18.- Subsecretaría de Salud Pública-Ministerio de Salud de Chile. Ordinario B27 N 1389 del 6 de mayo 2020 Recomendaciones para la vacunación escolar extramural durante la pandemia COVID-19 [Internet]. 2020. Available from: https://vacunas.minsal.cl/ wp-content/uploads/2020/07/Ord-B27-N ${ }^{\circ}$ 1389-Recomendaciones-para-la-vacunacionescolar-extramular-durante-pandemia-covid196-mayo-2020.pdf.
19.- Subsecretaría de Salud Pública-Ministerio de Salud de Chile. Ordinario B27 $\mathrm{N}^{\circ}$ 136029 abril 2020 Recomendaciones para la vacunación programática durante pandemia COVID-19 [Internet]. 2020. Available from: https://vacunas.minsal.cl/ wp-content/uploads/2020/07/Ord-B27-N ${ }^{\circ}$ 1360-Recomendaciones-para-vacunacionprogramatica-durante-pandemia-covid19-29abril-2020.pdf\%0A\%0A.

20.- Bastías M, Avendaño M, Muñoz F, Brstilo I, Álvarez A, Burgos P, et al. Campaña influenza 2020 en el contexto de pandemia por SARSCoV-2: una experiencia inédita de salud pública en Chile. Rev Chilena Infectol 2021; 38 (2): 178-84. doi. 